\title{
Weigh-in-motion (WIM) sensor response model using pavement stress and deflection
}

\author{
Gustavo Garcia Otto $^{\mathrm{a}, *}$, Jean-Michel Simonin ${ }^{\mathrm{b}}$, Jean-Michel Piau ${ }^{\mathrm{b}}$, Louis-Marie Cottineau ${ }^{\mathrm{b}}$, Olivier Chupin ${ }^{\mathrm{b}}$, \\ Leto Momm ${ }^{\mathrm{a}}$, Amir Mattar Valente ${ }^{\mathrm{a}}$
}

${ }^{\text {a }}$ Federal University of Santa Catarina, Brazil

${ }^{\mathrm{b}}$ French Institute of Science and Technology for Transport, Development and Network, France

This article aims at modeling the WIM sensor electrical behavior to provide a better understanding of the mechanical and electrical proprieties of piezoelectric sensors. The model is considered in the frequency domain, supposing any sinusoidal solicitation with constant frequency or any other type of solicitation. Tests, having real WIM sensors placed on the pavement of a test track at IFSTTAR/ Nantes was used to verify the nature of the electro-mechanical (EM) behavior of the real sensors over punching, flexural and extension effects. Also, two different pavement numerical simulations deliver the mechanical response using linear elasticity and viscoelastic proprieties. The output of these two simulations are compared with two WIM signals obtained at same load and speed at two different temperature conditions.

\author{
Keywords: \\ Weigh-in-Motion \\ WIM sensor technologies \\ WIM sensor test \\ Piezoelectric sensors \\ WIM accuracy \\ Pavement \\ Elasticity \\ Viscoelasticity \\ Road operation
}

\section{Introduction}

Weigh-in-motion (WIM) technology is one of the main tools for pavement management. It can provide an accurate description of the traffic on road network recording class of vehicles (trucks, cars and motorcycles by the weight, number of axle, distance of axle and size), total gross weight and axle weight (sometimes, weight per wheel), speed, date and time of each event. WIM have potential to minimize the problems of overloading practice. Overload trucks pose serious threats to road transport operations, with increased risks for road users, deterioration of road safety, severe impacts on the durability of infrastructure (pavements and bridges), and fair competition between transport modes and operations [1]. In addition, traffic counting data are an important input of pavement management systems for anticipating the evolution of pavement damage and the need for maintenance.

WIM systems were introduced in the United States in the mid1950s. First sensors used as scales were instrumented plates fixed in a frame mounted on the road, called bending plate scales. In the 80 's, a new generation of WIM sensors was developed using piezoelectric technology to infer wheel forces by the electric charge produced. Since then, many developments and progress have taken place, while sensors and techniques have been introduced and implemented.

Early researches show that piezoelectric sensors could be one of the solutions for measuring axle loads and for weigh-in-motion applications [2]. The piezoelectric effect is a phenomenon whereby mechanical energy is converted to electrical energy (and vice versa) within certain insulating materials. WIM sensors are generally installed in the pavement surface course and made flush to the pavement surface. The sensors respond to changes of loads with time rather than loads themselves. The weight of axle loads is generally calculated by integrating the output with time and by multiplying the integral by the speed of the vehicle and a calibration factor [3].

Two major studies about WIM systems and sensors were undertaken in the 90's. The COST323 project aimed to gather information on WIM technologies, to develop and specify European techniques, to define data base protocol and publish recommendations concerning WIM applications [4]. In the same period, the WAVE project (Weigh-in-motion of Axles and Vehicles for Europe) objectives were to improve WIM accuracy and load measurements on pavements and bridges, to provide reliable information to management systems and to develop calibration methods suitable to all European climates [5].

Not all WIM sensors are made with piezoelectric materials but they work similarly, transforming the stresses induced by the vehicle in motion into an electrical response. Today, WIM strip sensors for pavement applications can be found based on quartz crystal, ceramics, polymers, or also electric strain gauges or optical fibers technologies. Some of their characteristics are presented below:

- The strip sensors with quartz technology are delivered as bars. The external part is an aluminum profile and the internal is filled with pastilles of quartz regularly spaced along the bar. They are mechanically independent one from each other, but are connected to the same conductive element to deliver the electric output. The internal shape is designed in such way that only vertical load stimulates the internal pastilles of quartz [6].

- The ceramic sensors are made of a powder ceramic compressed inside a metallic profile (some types are design as a tube with a circular compressed shape). A central core made of copper, connected to the output, collects the electric charge generated by the stress in ceramic element [7].

- The polymer strip sensor is composed by a polymeric film, made of PolyVinyliDene Fluoride (PVDF) spiral-wrapped inside of a copper flat tube. When load is over the sensor, compression acts on the tube which transmits to the polymer, an electrical charge is then transmitted to output [8].

- The optical sensor is designed with an optic fiber merged into a dense foam. The optical principles of measurement can be either based on polarimetry interpretation or in the Michelson interferometer. The components of the interferometer assess the fringe number and fringe period to approximate the load applied over the sensor [9]. In the polarimetric sensor, the light from a laser diode is polarized by a polarizing beam splitter and launched into a high birefringence transmission fiber, in such way, that only one polarization mode is excited [10]. An optical-electronic interface detects the changes in the optical signal and transforms them into electric signals for traffic data processing.

- The strain gauge strip sensors use strain gauges inside of a metallic profile. The WIM system correlates the strain measurement with the corresponding axle load value [11].

There are other WIM sensor technologies which has different response mechanism. For those cases, the electro-mechanical model does not apply.

In Brazil, the DNIT has started a national weigh-in-motion project in 2007 to evaluate WIM applications to Brazilian conditions of pavement and traffic. Two type of tests were performed, one concerning WIM accuracy and performance and a second about load damage on pavement structure. A test site was built near the city of Araranguá (Santa Catarina state, Brazil, $28^{\circ} 58^{\prime} 40.28^{\prime \prime}$, $49^{\circ} 32^{\prime} 4.84^{\prime \prime} \mathrm{W}$ ), near to the DNIT weigh station. There are four groups of sixteen WIM sensors (piezoelectric ceramics, polymer, 
quartz and optical technology) and one of pavement evaluation sensors (strain, pressure and temperature) on the test site. Tests are performed comparing dynamic weight measurements with pre-weighed values.

In France, IFSTTAR started a national WIM project in 2014 for direct enforcement of heavy vehicles. The project was organized in six tasks, related to: sensor and pavement interaction, multiple sensors WIM, bridge WIM, on site test, and feasibility of OIML R134 type approval, expert study. The experimental campaign aims at comparing different WIM sensor technologies in controlled condition in addition to the laboratory tests. The pavement testing facility on IFSTTAR Nantes is a full-scale facility, designed to study the damage caused by axle load of a real pavement. It is a circular test track of $120 \mathrm{~m}$ long and $6 \mathrm{~m}$ wide, four loading arms, with a maximum load level of $130 \mathrm{kN}$ on each arm (65 kN on single load or dual axles) and a maximum speed of $100 \mathrm{~km} / \mathrm{h}$. On the test track pavement, there are three technologies of WIM sensors (piezoelectric ceramics, polymer and quartz). The experimental campaign is designed to study the repeatability of WIM sensors under different conditions of loading, tire pression, temperature, lateral position, speed and axel configuration.

This article aims at proposing an electro-mechanical model for WIM sensors to investigate and analyze their interaction with the road pavement under a known loading and two different temperature conditions.

\section{Electro-mechanical (EM) model for WIM sensors}

\subsection{Model including the effect of sensors deformation due to pavement deflection}

In the following, we consider WIM devices as beams, that we note $\left(B_{S}\right)$ for a nude sensor (subscript $S=$ sensor) and $\left(B_{P}\right)$ for a sensor embedded in a pavement (subscript $P=$ pavement). In that case $\left(B_{P}\right)$ represents not only the sensor itself but also the wrapping resin filling the trench done at the pavement surface. The geometrical and mechanical proprieties of $\left(B_{S}\right)$ and $\left(B_{P}\right)$ may be widely different. In both cases, we note $s$ the longitudinal coordinate along the beam, $z$ the coordinate along the vertical axis, $u(s), w(s)$ the longitudinal and vertical displacements of the WIM sensor along its neutral fiber. Depending upon the sensor fabrics (material and composition elements), the beam $\left(B_{S}\right)$ can be considered as mechanically and electrically homogeneous or heterogeneous along its longitudinal direction. Nevertheless, in this last case we consider the internal structure of $\left(B_{S}\right)$ sufficiently short compared to the width of the tire imprints, to make it possible to homogenize its EM response with $s$. But possible its local EM properties may be dependent on $s$.

Before writing a model for the EM response of WIM sensors, let us recall that pavements are multi-layered structures, which undergo more, or less, important deflection under the passing of vehicles, depending upon the materials making the pavement, the layer thickness and the soil bearing capacity [12].

When installed in the pavement, the WIM sensors respond mechanically to two types of effects under the traffic load (see Fig. 1).

The first one, which WIM sensor are supposed to be especially sensitive, is the local stress redistribution within the WIM section located close and below the tire imprints, nominated as "primary" EM response. For a homogeneous beam, such a stress distribution would be mostly characterized by vertical stresses (plus possible horizontal confining stress depending upon the sensor fabrics and wrapping). To give a precise definition to this component of the EM response of WIM devices, we consider it in the following as the one that would arise if $\left(B_{S}\right)$ or $\left(B_{P}\right)$ were relying on a rigid support without deformation, that is for $w(s)=0$ (and then also $(s)=0$, at least at points $s$ far from the tire imprint).

The second mechanical effect, which WIM sensors are submitted to the flexural deformation resulting from the pavement deflection due to wheel loads, not only those passing on the WIM sensor itself, but also the ones located in its vicinity. WIM devices are supposed to be only weakly dependent if not at all, of this effect, but since it is part of our objectives to check this assumption, we will also consider this action in our model. At the opposite of the "primary" EM response of WIM sensors, this component will be supposed to be directly linked to $w(s)$ and $u(s)$ displacements.

Beside for a general model of WIM devices, we will consider their response in the frequency domain, which makes it easy to account for both:

- A possible viscoelastic mechanical behavior of sensors (especially when considering wrapped sensors $\left(B_{P}\right)$ with resin),

- Electric signal, not necessarily in phase with the load.

Thus, considering only the case of additive and linear effects, we propose to describe the EM response of WIM sensors by the general equation:

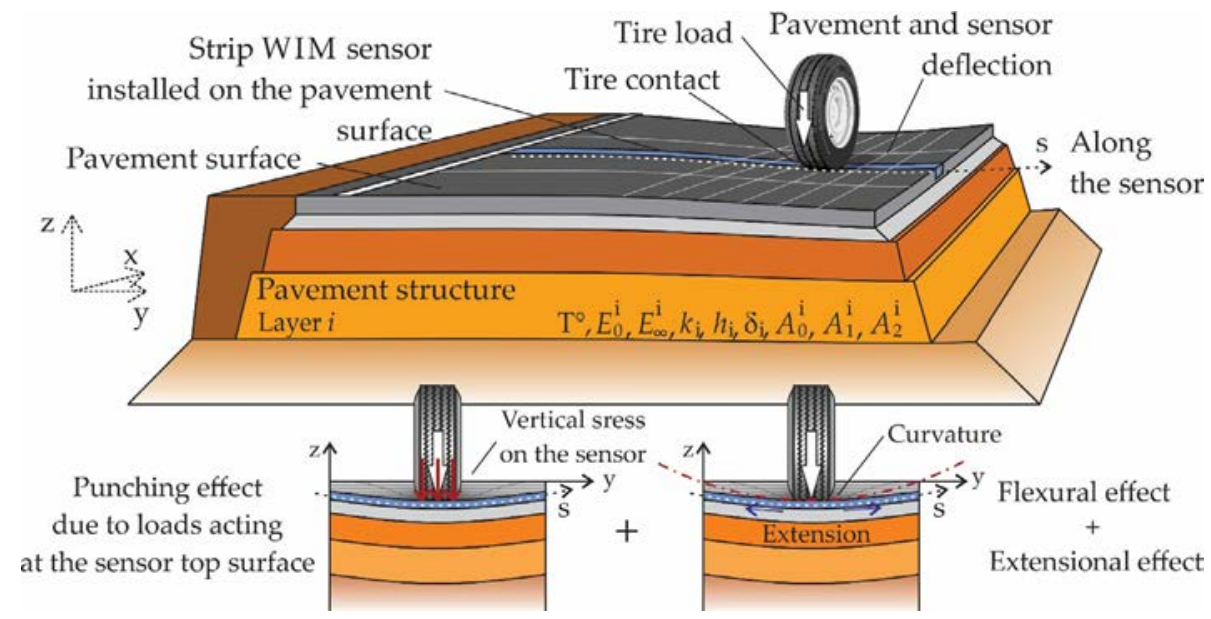

Fig. 1. WIM sensor response based on pavement deflection and stress transmitted to the WIM sensor under a tire load influence. 


$$
\begin{aligned}
Q^{*}(\omega, \mathrm{T})= & \underbrace{\int_{0}^{l} \alpha^{*}(s, \omega, T) p^{*}(s, \omega) d s}_{\begin{array}{c}
\text { Primary punching effect } \\
\text { due to loads acting at } \\
\text { the sensor top surface }
\end{array}}+\underbrace{\int_{0}^{l} \beta^{*}(s, \omega, T) C^{*}(s, \omega, T) d s}_{\text {Flexural effect }} \\
& +\underbrace{}_{\begin{array}{l}
\text { Extensional effect } \\
\int_{0}^{l} \gamma^{*}(s, \omega, T) u^{\prime *}(s, \omega, T) d s
\end{array}}
\end{aligned}
$$

With:

$Q^{*}(\omega, T)$ : complex amplitude of the electric tension $V$ at pulsation $\omega$ and temperature $T$, output of the charge amplifier (sensibility transfer factor $V / p C$ ) which WIM piezoelectric sensors is connected (sensitivity transfer factor $p C / N$ ),

$p^{*}(s, \omega)$ : complex amplitude if the pressure exerted at the top surface of the sensor, as a function of abscissa $s$ and pulsation $\omega$,

$C^{*}(s, \omega, T)=\frac{d^{2} w^{*}(s, \omega)}{d s^{2}}$ : complex amplitude of the WIM sensor curvature,

$u^{\prime *}(s, \omega, T)=\frac{d u^{*}(s, \omega)}{d s}$ : complex amplitude of the WIM sensor extension.

$\alpha^{*}(s, \omega, T), \beta^{*}(s, \omega, T), \gamma^{*}(s, \omega, T)$ : respectively, sensitivity functions of WIM sensors to the primary punching effect $(V / k N$, $V$ - volts; $k N$ - kilo Newton), deflection $(V / m, m$ - meters) and extension $(V / m)$. These functions may be also dependent on temperature.

The charge amplifier converts the electrical charge from the piezoelectric sensors to output voltage $Q^{*}[V]=$ force $[N]$ on the sensor $\times$ sensor sensitivity $[p C / N] \times$ charge amplifier transformation factor $[V / p C]$.

It must be noted that the use of the second and first derivatives of $w^{*}$ and $u^{*}$ in (1) eliminates any influence of rigid body motions in the EM response of WIM sensors, as expected. In a real situation, WIM sensor will be attached to the pavement. The sensor body and the pavement will experience the same mechanical deflection.

The response of WIM sensors in the time domain can be derived from (1) using the usual framework of Fourier's transformation.

Using different types of WIM sensors technology, the model parameters can be calibrated to represent their response once in embedded in pavement. Among WIM sensors, the piezoelectric and optical sensors have more close response to load and deflection effects. In those cases, the electrical response can be obtained using the EM model and the pavement mechanical reactions when both, sensor and pavement, are submitted to a moving axle load.

\subsection{Laboratory approach to identify the coefficients of sensitivity of the model}

The coefficients of sensitivity of the EM model, in the Eq. (1) above, can be identify by two types of laboratory tests, pure punching and flexural. Usually, sensor can be tested in laboratory only in nude state, without wrapping material. A punctual sinusoidal compressive force $F(t)$, applied at abscissa $s^{0}$ for the time t, will generate a pure flexural state without extension at the neutral fiber $(u=0)$.

Then writing,

$F(t, s)=F^{*}(a-\cos \omega t) \delta\left(s^{0}\right)$

With $a>1$ and discarding the constant component $\alpha^{*} F^{*}$ (which does not give detectable electrical effect on the frequency domain), the previous model writes:

$Q^{*}(\omega)=\alpha^{*}\left(s^{0}, \omega, T\right) F^{*}+\int_{0}^{l} \beta^{*}(s, \omega, T) C^{*}(s, \omega) d s$
With:

$F^{*}$ : force amplitude, here consider as real and positive for compression,

$\delta\left(s^{0}\right)$ : spatial Dirac distribution at $s=s^{0}$.

To find the two sensitivity functions $\alpha^{*}$ and $\beta^{*}$, two types of test can be performed, depending on the beam support:

1) Pure punching test with the sensor-beam $\left(B_{s}\right)$ is on a flat continuous and very rigid support. With the curvature $C^{*}$ being equal to zero, the model simplifies to:

$Q^{*}(\omega)=\alpha^{*}\left(s^{0}, \omega, T\right) F^{*}$

2) Three-point bending tests with different spans of support to check whole model and identify also the function $\beta^{*}$.

In all cases, the amplitudes $Q^{*}$ and $F^{*}$ need to be recorded. In the second case, the cyclic beam deflection must be measured using displacement sensors during the same time $t$.

To simplify, $\beta^{*}$ is supposed to be independent of the abscissa $s$. Then considering the relationship between curvature and deflection and with $u=0$, the model writes in such test:

$Q^{*}(\omega)=\alpha^{*}\left(s^{0}, \omega, T\right) F^{*}+\beta^{*}(\omega, T)\left(\frac{d w^{*}}{d s}(l, \omega)-\frac{d w^{*}}{d s}(0, \omega)\right)$

Furthermore, assuming a linear mechanical behavior of the beam, a geometrical factor $\varphi_{i}^{*}$ can be determined for each span $(i=1,2, \ldots)$, possibly depending upon $\omega$ due to inertial forces (rather than viscoelastic in case of nude sensors), such as:

$\frac{\partial w_{i}^{*}}{\partial s}(l, \omega)-\frac{\partial w_{i}^{*}}{\partial x}(0, \omega)=\varphi_{i}^{*}(\omega) F^{*}$

Then, for each tested combination (load amplitude and location, frequency, $\operatorname{span} n^{\circ} i$ ), one obtains a relationship with shape:

$Q_{i}^{*}=\left(\alpha^{*}\left(s^{0}\right)+\beta^{*} \varphi_{i}^{*}\right) F_{i}^{*}$

Note also that varying the temperature (with the use of a climatic chamber, for instance) would make it possible to study the effect of this parameter on the response of sensors. At the laboratory test, the temperature $T$ is constant for the different test modalities (span conditions and type of support).

Now, for given values of $s^{0}, \omega, F_{i}^{*}$, the two unknowns $\alpha^{*}\left(s^{0}\right), \beta^{*}$ can be derived from Eqs. (3) and (7) and from the measurements of $Q_{i}^{*}$ and $\varphi_{i}^{*}$. For instance, in case of tests performed for two span conditions, on gets:

$\alpha^{*}=\frac{1}{\varphi_{1}^{*}-\varphi_{2}^{*}}\left(-\varphi_{1}^{*} \frac{Q_{2}^{*}}{F_{2}^{*}}+\varphi_{2}^{*} \frac{Q_{1}^{*}}{F_{1}^{*}}\right)$

$\beta^{*}=\frac{1}{\varphi_{2}^{*}-\varphi_{1}^{*}}\left(\frac{Q_{2}^{*}}{F_{2}^{*}}-\frac{Q_{1}^{*}}{F_{1}^{*}}\right)$

In the case of tests with multiples span values, leading to an overdetermined linear system, $\alpha^{*}$ and $\beta^{*}$ can be solved using the least square method and the redundancy of data uses do estimate the accuracy of the model.

The relative importance of terms $\beta^{*}, \gamma^{*}$ versus $\alpha^{*}$ on the response of a WIM sensor embedded in a pavement can also be directly estimated from in situ measurements. Using, for instance, a metal plate covering the sensor trench at the same time without contact with the sensitive surface. Then, it is possible to compare the "normal" response of the sensor submitted to a given load (rolling wheel loads or FWD load) with the response measured with the metallic plate for the same loading conditions. 
Obtaining a significant signal in this last case would be the sign for a sensor sensitive to pavement deformation.

\subsection{Pavement mechanical approach to obtain EM sensor response}

The EM model couple the force and deflection acting on the sensors to produce a proportional WIM sensor electrical response $Q^{*}$. In this section, we propose represent the pavement mechanical behavior model to obtain the piezoelectric WIM sensor response. A pavement mechanical software provides the strains and stress on multiples layers of a semi-infinity body according to the mechanical characteristics of the material. It will be valid saying that the sensor deforms at the same rate and magnitude as the pavement. The methodology for this approach consist to compute the pavement strain and stress response along the as specific set of coordinates $(x, y, z)$. With mathematic adjustment, the strain and stress matrix obtained can be transferred to the EM model as input and the electrical response $Q^{*}$ can be calculated.

The sensor is a bar with a total length $l$ and a square crosssection with equal sides length of $h$. Piezoelectric proprieties are constant and homogeneous. Installation alignment follows the $y$ ordinate. The tire passes transversally to the sensor in the same that is found in a real WIM installation. For this analysis, tire travels over the abscissa $x$, at constant speed and without deviating the initial position. The sensor is considering to be at the position $x=0$.

The electrical response $Q^{*}$ of the WIM sensor, when installed in the pavement, depends on the sum of all force $\sum_{0}^{l} F^{*}$ along the sensor surfaces and on the difference of slope $\Delta P\left(\frac{d w^{*}}{d s}(l)-\frac{d w^{*}}{d s}(0)\right)$ at the extremities of the sensor bar. Since sensor and pavement deforms together, the difference of slopes of the pavement at the same local of the sensor extremities will be the input for the model.

$Q^{*}=\alpha^{*} \sum_{0}^{l}\left(F^{*}\right)+\beta^{*}\left(\frac{d \mathrm{w}^{*}}{d s}(l)-\frac{d w^{*}}{d s}(0)\right)$

The output $Q^{*}$, for each position $x$ of the tire, is a function of the acting forces and the sensor curvature along the surfaces of the sensor. The reacting forces provide by the pavement software are the input forces to the model. $F(x)$ will be proportional to the sum of the horizontal and vertical reacting forces distributed over the sensor surfaces, $F_{x x}(x)$ and $F_{z z}(x)$ respectively. $F_{x x}$ results from the stress $\sigma_{x x}$ acting on the two surfaces at the plane $(y, z), \Delta y$ is the steps between each point at y axle.

$F_{x x}=h \cdot \sum_{l_{\text {sensor }}} \sigma_{x x}(y) \cdot \Delta y$

At each position of the tire, in $x$, the forces $F_{x x}(x)$ acting along the two surfaces is:

$\left[F_{x x}\right](x)=\left[h \cdot \sum_{l_{\text {sensor }}} \sigma_{x x}(y) \cdot \Delta y\right](x)$

$F_{z z}$ results from the stress $\sigma_{z z}$ acting on the two surfaces at the plane $(x, y)$.

$F_{z z}=h \cdot \sum_{l_{\text {sensor }}} \sigma_{z z}(y) \cdot \Delta y$

In the same way, $F_{z z}(x)$ acting along the sensor is:

$\left[F_{z z}\right](x)=\left[h \cdot \sum_{l_{\text {sensor }}} \sigma_{z z}(y) \cdot \Delta y\right](x)$

The difference of slope $\Delta P$ of the extremities of the sensor $\left(l_{0}, l_{n}\right)$ is then calculated by the difference of slope of the deflection at the same point on the pavement.

$$
\begin{aligned}
\Delta P & =\left(\frac{\Delta \operatorname{Def}}{\Delta s}\left(l_{n}\right)-\frac{\Delta \operatorname{Def}}{\Delta s}\left(l_{0}\right)\right) \\
& =\left(\frac{\operatorname{Def}\left(l_{n}\right)-\operatorname{Def}\left(l_{(n-1)}\right)}{s_{n}-s_{(n-1)}}-\frac{\operatorname{Def}\left(l_{1}\right)-\operatorname{Def}\left(l_{0}\right)}{s_{1}-s_{0}}\right)
\end{aligned}
$$

At each position of the tire, in $x$, we then can calculate $\Delta P(x)$ :

$[\Delta P](x)=\left[\frac{\Delta D e f}{\Delta s}\left(l_{n}\right)-\frac{\Delta D e f}{\Delta s}\left(l_{0}\right)\right](x)$

The EM model couple the forces acting on the sensor surfaces with the curvature by the two coefficients $\alpha^{*}$ and $\beta^{*}$ as a tire passes over the region of the sensor.

$Q(x)=\alpha^{*} \cdot\left(F_{x x}(x)+F_{z z}(x)\right)+\beta^{*} \cdot \Delta P(x)$

In the next part, the electrical response $Q(x)$ of a known WIM sensor using ALIZE-LCPC and ViscoRoute. Both software provides the mechanical response on the layers of several types of pavement structure using mechanical parameters:

- ALIZE delivers the stresses and strains generated by a traffic load into a pavement structure by a module named "ALIZEmechanical module" [13]. The ALIZE-LCPC which is used in the French design of pavements. ALIZE-LCPC computes the Brumister solution [14] of an elastic multilayered half-space under static loading.

- ViscoRoute [15] provides a time-domain model for pavement response under moving dynamic load which takes explicitly into account the time-dependency of the load within its influence area at any particular location. The algorithm integrates the viscoelasticity behavior of asphalt materials through the Huet-Sayegh model [16,17]. It is particularly well-suited for the modelling of asphalt overlays [18]. The moving load effects and the thermo-viscoelastic behavior of asphalt materials are accounted to represent the behavior of flexible pavements for different conditions of temperature and vehicle speed.

\section{Real WIM sensor electrical response and EM model comparison}

This section aims at comparing the real piezoelectric WIM sensor electrical responses, obtained during the experimental campaign at the IFSTTAR carousel, and the EM model response of a pavement response model obtained by both pavement software. The load carried by the tire and the pavement characteristics are the same found at the test site, such as: the total load, transversal

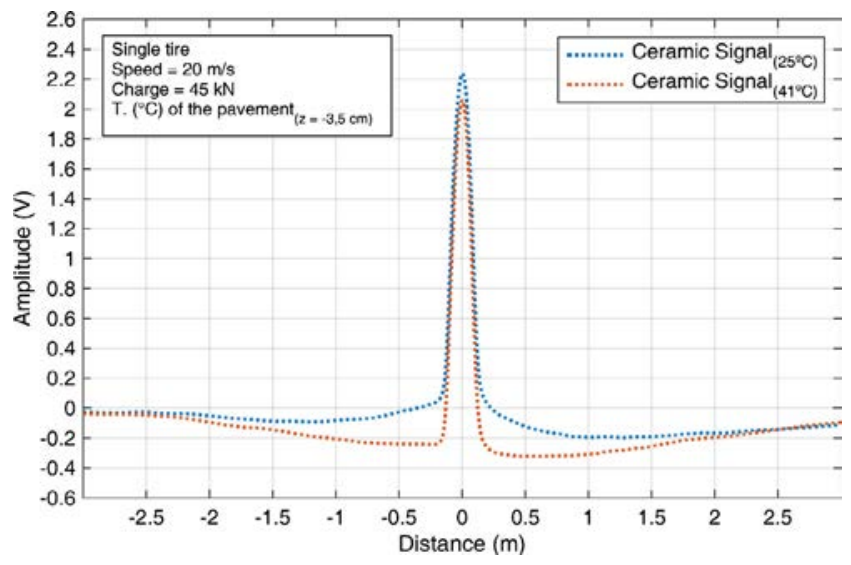

Fig. 2. Real piezoelectric ceramic sensor response under an influence of a single tire loaded with $45 \mathrm{kN}$, passing at $20 \mathrm{~m} / \mathrm{s}$, at different pavement temperature conditions $\left(25^{\circ} \mathrm{C}\right.$ and $\left.41^{\circ} \mathrm{C}\right)$. 
position (regarding the sensor surface), speed, pavement structure and temperature changes. This section will verify the variation of the electrical response of a WIM piezoelectric ceramic sensor and the coefficients $\alpha^{*}$ and $\beta^{*}$ of the model using the elastic and viscoelastic pavement models. With the second model, a variation of temperature is imposed to obtain a different response of the pavement and, consequently, a different response of the EM model.

\subsection{Real WIM sensor electrical response}

Fig. 2 shows two signals from the same ceramic sensor installed in the carousel test track. Both signals results from a passage of a single tire loaded with $45 \mathrm{kN}$. The speed is constant and equal to $20 \mathrm{~m} / \mathrm{s}$. The blue dotted line corresponds to a signal acquired when the temperature of the pavement was $25^{\circ} \mathrm{C}$, at $\mathrm{z}=-3.5 \mathrm{~cm}$ from the surface, the red dotted line corresponds to a signal acquired when temperature at the same depth was $41^{\circ} \mathrm{C}$. The ceramic sensor is highly sensitive to temperature variation, this difference of sixteen degrees is sufficient to change their electrical response. Indicates that a correction factor regarding temperature must be considered for high accuracy.

Test track is circular and the pavement evenness is constant as the tire travels over a circuit, in these conditions, the signals obtained from the ceramic sensors shows good repeatability. The smalls variations (ripples along the signal) are present in all signal acquired, this can be explained due to fluctuations of the dynamic load of the body load transmitted to the tire. The ceramic sensor reacts to the strain and stress generated all around the pavement.

The region near the signal central peak describes what happen at the moment that the tire passes directly over the sensor, called tire footprint. The distance between the moment that the tires touch it until the moment that tires leaves is about the same size of the footprint. Consequently, the area under the signal is proportional to the load transmitted to the sensor [19]. The magnitude of the central pick and the shape of the signal varies as the temperature changes, indicating that the ceramic sensor is sensitive to temperature. The pavement deflection has direct effect on the sensor response, the sensor responds to the load even before the tire touches it. This sensibility due to deflection happens at six meters before and it goes farther after the tire leaves the sensor, concluding that the ceramic sensor responds electrically as the pavement deflect.

\subsection{Pavement mechanical parameters}

The pavement of the fatigue test track is composed by different layers, which three of them are bituminous materials and one is a granular soil. The input data for the pavements model are layer thickness, elastic modulus, type of material, type of interaction between layers and the output are the internal stress and deflection. Table 1 gives the properties of the pavement structure layers used as input data in the numerical analysis using ALIZE and ViscoRoute. The materials proprieties are approximations of the material proprieties in the standard NF P98-086 [20]. The depth of each layer is known from the pavement construction. The structure is composed by a top layer of $0.08 \mathrm{~m}$ made of an asphalt concrete
Béton Bitumineux Semi-Grenu (AC-BBSG), by one base and subbase layers with asphalt concrete of Grave Bitume type 3 (AC-GB3) of $0.13 \mathrm{~m}$ each, and by a pavement foundation type 2 (PF2) [12].

For each layer, Poisson's ratio is equal to 0.35 and the density is equal to $2100 \mathrm{~kg} / \mathrm{m}^{3}$. The three first layers are considered as viscoelastic (see Table 2 for the Huet-Sayegh parameters). Temperature for the viscoelastic model is fixed, in each interaction, at one of the two temperatures $\left(25^{\circ} \mathrm{C}\right.$ and $\left.41^{\circ} \mathrm{C}\right)$, for all bituminous layers. The reference load is $45 \mathrm{kN}$ and it passes over the pavement at a constant speed of $20 \mathrm{~m} / \mathrm{s}$.

\subsection{Comparison between EM model and real signal}

Fig. 3 show the comparison between the ceramic sensor signal and the result obtained from the EM model using ALIZE. The dotted red line is the ceramic signal obtained when temperature of the pavement was $41^{\circ} \mathrm{C}$, the purple solid line is the EM model $Q(x)_{\text {(ElasticModel) }}$. The signal $Q(x)$ is symmetric with respect to the origin $x=0$, mainly because in the Brumister solution the load is static centered in a half space. In $Q(x)$, the effect of the load distribution over the sensor surface $F_{z z}$ doesn't change in comparison with the ceramic signal. The effect of curvature $\Delta P$ cannot be neglected, the main difference between signals is the viscoelastic behavior of the pavement structure. The two coefficients of the EM model that best fits $Q(x)$ to the shape of the real signal are $\alpha^{*}=0.45 \mathrm{~V} / \mathrm{kN}$ and $\beta^{*}=-2300 \mathrm{~V} \cdot \mathrm{m}$.

Considering this specific ceramic sensor signal, a viscoelastic pavement model must be used to represent the exact effect of the load and deflection. However, the use of any computational tool based on pavement elastic model cannot be discarded, it can be perfectly applied for the case when the structure is a concrete pavement for example.

Fig. 4 show the comparison between ceramic signal with the result obtained with the EM model $Q(x)$ using ViscoRoute. Again, the dotted red line is the ceramic signal obtained when temperature of the pavement was $41^{\circ} \mathrm{C}$, but the purple solid line is the EM model $Q(x)_{(\text {Viscolelastic Model) }}$ whit the parameters of the pavement structure considering a temperature of $41^{\circ} \mathrm{C}$. This time, the shape of $Q(x)$ is closer to the shape of the ceramic signal. $\Delta P$ and $F_{x x}$ gives the effect of viscoelasticity to the EM model. The two coefficients needed to best fit the EM model $Q(x)$, in comparison to the shape of the real signal, are $\alpha^{*}=0.46 \mathrm{~V} / \mathrm{kN}$ and $\beta^{*}=-1700 \mathrm{~V} \cdot \mathrm{m}$.

The superposition is lees apparent after $x=1$, it appears that the real signal is more time dependent then the EM model predicts. The difference indicates that the real sensor piezoelectric material generates electric charge much more longer then the pavement retains the deformed structure. The small variation, ripples, observed between the two curves could be explained by the fluctuation of the tire load transmitted to the pavement.

Fig. 5 show comparison the changes of temperature over the ceramic sensor and the result obtained with the EM model $Q(x)$, both using ViscoRoute. In blue, the dotted line represents the ceramic sensor real signal and the solid line represents $Q(x)$ when temperature of the pavement is around $25^{\circ} \mathrm{C}$. In red, the dotted and solid lines represent the real signal and $Q(x)$ respectively when temperature of the pavement is $41^{\circ} \mathrm{C}$.

Table 1

Properties of the pavement structure layers, input data considered in the numerical analysis using ALIZE and ViscoRoute.

\begin{tabular}{|c|c|c|c|c|}
\hline Structure & Thickness (m) & Modulus (MPa) & Type of material & Type of interactions \\
\hline Wearing course & 0.08 & 7000 & AC-BBSG3 & - \\
\hline Base course & 0.13 & 9000 & AC-GB3 & Bonded \\
\hline Subbase course & 0.13 & 9000 & AC-GB3 & Bonded \\
\hline Subgrade & Infinite & 50 & PF2 & Bonded \\
\hline
\end{tabular}


Table 2

Huet-Sayegh parameters for the BBSG and the GB layer of the pavement structure, input data considered in the viscoelastic numerical analysis using ViscoRoute [18].

\begin{tabular}{|c|c|c|c|c|c|c|c|c|}
\hline Type of material & $\mathrm{E}_{0}(\mathrm{MPa})$ & $\mathrm{E}_{\infty}(\mathrm{MPa})$ & $\delta$ & $\mathrm{k}$ & h & A0 & A1 & $\mathrm{A} 2$ \\
\hline BBSG & 18 & 40995 & 2.356 & 0.186 & 0.515 & 2.2387 & -0.3996 & 0.00152 \\
\hline GB & 31 & 38814 & 1.872 & 0.178 & 0.497 & 2.5320 & -0.3994 & 0.00175 \\
\hline
\end{tabular}

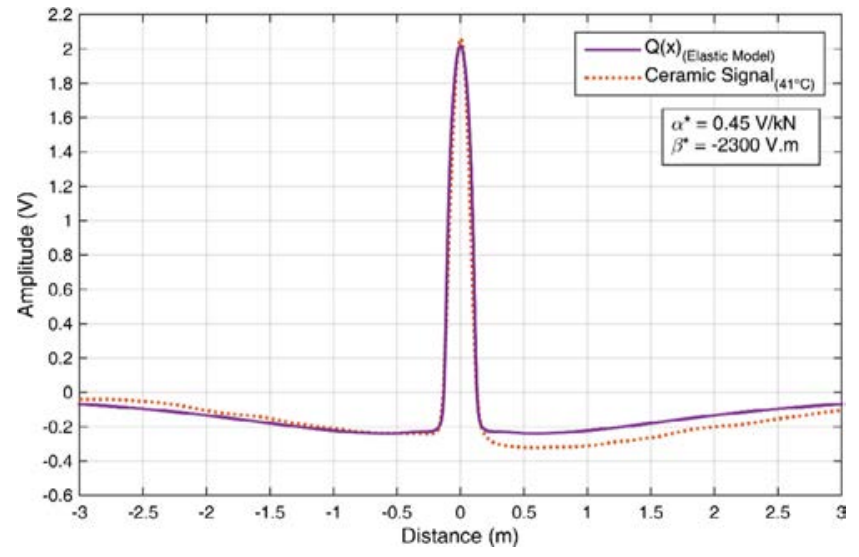

Fig. 3. Comparison between the sensor EM model $Q(x)_{(\text {ElasticModel })}$ and the real ceramic signal under a load of $45 \mathrm{kN}$, constant speed of $20 \mathrm{~m} / \mathrm{s}$ and pavement temperature of $41^{\circ} \mathrm{C}$.

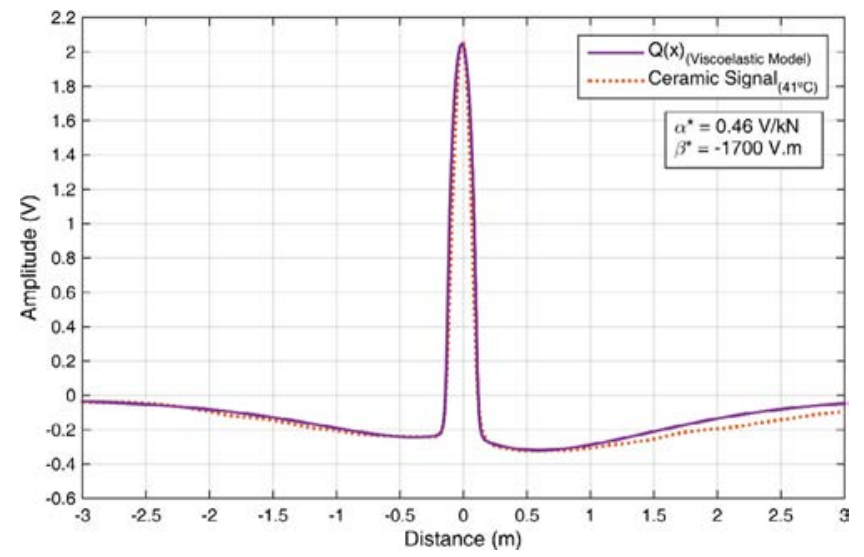

Fig. 4. Comparison between the sensor EM model $Q(x)_{(\text {ViscoelasticModel })}$ and the real ceramic signal under a load of $45 \mathrm{kN}$, constant speed of $20 \mathrm{~m} / \mathrm{s}$ and pavement temperature of $41^{\circ} \mathrm{C}$.

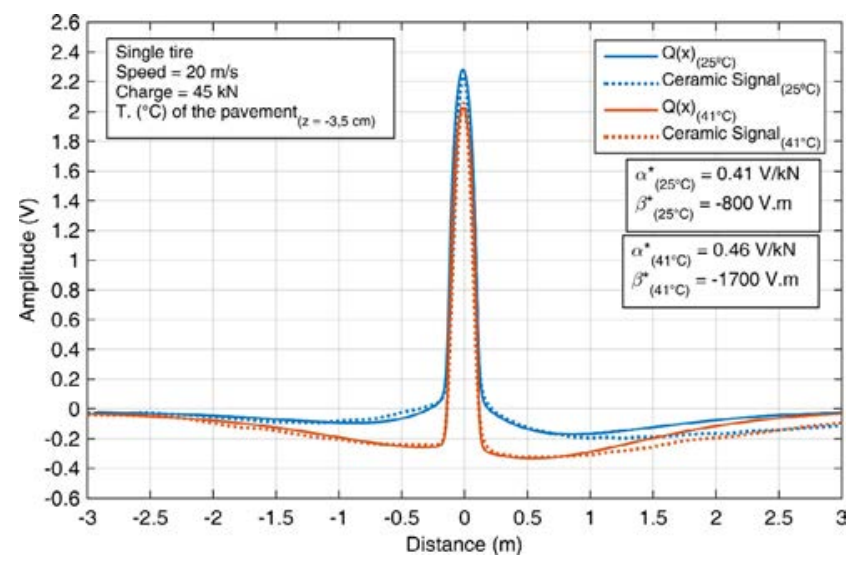

Fig. 5. Comparison between the sensor EM model $Q(x)_{\left(V_{\text {ViscoelasticModel })}\right.}$ and the real ceramic signal considering change of pavement temperatures of $25^{\circ} \mathrm{C}-41^{\circ} \mathrm{C}$.
Both signals results from the same load which produce the same impulse over the surface of the sensor, $F_{z z}$ constant. The difference between the picks results manly from the changing of in each temperature, were $\alpha^{*}$ is the sensitivity functions of WIM sensors to the primary punching effect. The effect of curvature $\Delta P$ varies because the curvature of the pavement changes according to the temperature.

The coefficient $\alpha_{\left(41^{\circ} \mathrm{C}\right)}^{*}$ is approximatively $0.46 \mathrm{~V} / \mathrm{kN}$ at $41^{\circ} \mathrm{C}$, when temperature drops to $25^{\circ} \mathrm{C}, \alpha_{\left(25^{\circ} \mathrm{C}\right)}^{*}$ drops approximatively to $0.41 \mathrm{~V} / \mathrm{kN}$. The coefficient $\alpha^{*}$ varies about $11 \%$ between temperature. The coefficient related to deflection $\beta_{\left(41{ }^{\circ} \mathrm{C}\right)}^{*}$ is $-1700 \mathrm{~V} \cdot \mathrm{m}$

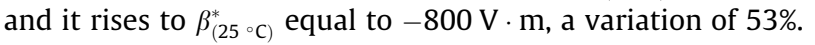

\section{Conclusions}

This article presented an Electro-mechanical model to compute the response of piezoelectric sensors under influence of a uniform load moving at constant speed, and static, and the pavement characteristics. Emphasis was put on the model the punching, flexion and extension effects so that the load and pavement conditions could be considered (and mixed) in the solution process.

A laboratory approach will permit to identify the two main coefficients of sensitivity that allows the EM model for any WIM sensor. The pure punching and three-point bending test can be used. The First test gives the value of the first coefficient $\alpha^{*}$ directly. The second test gives the two mains coefficients $\alpha^{*}$ and $\beta^{*}$ with results from two different distances between support.

The influence of the pavement could be tested using ALIE and ViscoRoute. First is based on the Brumister solution of elastic multilayered, second is based on algorithm that integrates the viscoelasticity behavior of asphalt materials through the Huet-Sayegh model. The output signals obtained with the pavement mechanical approach could be compared with two real ceramic signals at different temperature conditions.

The following concluding remarks are made: (i) The ceramic sensor signals obtained during the experimental campaign in Nantes show that the sensor responds to load and pavement deflection effects combined, also it depends to the temperature conditions. (ii) The electrical signal from the EM model using ALIZE presents he effect of the deflection of the pavement. It is different form the ceramic signal due to the viscoelastic characteristic of the pavement, which is transmitted to the sensor in the instant of weighing process. (iii) The EM model electrical response using ViscoRoute show similar response in comparison with the ceramic sensor, including temperature conditions. The response due to pavement deflection are $\alpha^{*}, \beta^{*}, \gamma^{*} \rightarrow(\omega, T, s)$ similar in shape and amplitude. Moreover, the sensor seams to retain electrical charge longer period than the deformed pavement structure. (iv) The comparison between signals obtained at different temperatures show significant dependence of the sensor to temperature condition. Moreover, viscoelastic behavior of the pavement could be represented in each temperature conditions. As expected, the sensitivity coefficients of the EM model changes according to temperature.

The model developed in this paper will allow, in future works, to introduce more complex sensitivity parameters () considering the WIM sensor characteristics and the interactions with different pavements types and different loading conditions. 


\section{Acknowledgments}

To professor Dr. Leto Momm, who has dedicated his lifework to pavement study. Authors acknowledge DGITM that funded the experiments at IFSTTAT and all IFSTTAR researchers and technical staff that worked on this project. Thanks to the MAST department, in specially Doctor Pierre Hornich, chief of the laboratory LAMES, and the staff group from the fatigue carousel at Nantes. Thanks to all team members from the COSYS. Authors acknowledge the CAPES, foundation of the minister of education in Brazil for providing the scholarship for PhD student Gustavo Otto, to the DNIT for funded the WIM project in Brazil and to the Federal University of Santa Catarina. Thanks to LabTrans team who has worked in the test track of Araranguá.

\section{References}

[1] B. Jacob, V.F. Beaumelle, Improving truck safety: Potential of weigh-in-motion technology, Elsevier, IATSS Res. 34 (2010) 9-15.

[2] D.J. Clark, A Comparison of Bending Plate and Piezoelectric Weigh-in-Motion Systems Master of Science Thesis, Montana State University Boezman, Montana, 2002.

[3] W.H. Newton, B.A. Frith, I.A. Barbour, Use of Weigh-in-Motion systems to aid the selection of goods vehicles for enforcement weighing, in: Proceedings 3rd International Symposium on Heavy Vehicle, U.K., 1992.

[4] COST 323, Weigh-in-Motion of Road Vehicles, Final Report, European WIM Specification, LCPC publication, Paris, 2005.

[5] B. Jacob, E. O'Brien, Weigh-in-motion of Axle and Vehicle for Europe (WAVE), Executive Summary, 4th Framework Programme, Transport (2000).

[6] KISTLER. Lineas Quartz Sensor for Weigh-in-Motion (WIM). https://www. kistler.com/br, 2016 (accessed 28.09.2016).
[7] Thermocoax, The piezo-electric sensor Vibracoax, http://www.termya.es/pdf/ sensores/otros sensores/SENSORES\%20PIEZOELECTRICOS.pdf, 2007 (accessed 13.07.2016)

[8] TE Connectivity, RoadTrax BL Traffic Sensor Piezoelectric Axle Sensor, Product Guide, http://www.te.com/content/dam/te-com/documents/sensors/global/ TE_SensorSolutions_RoadTrax-BL-Piezoelectric-Axel-Sensor_SS-TS-TE600.pdf, 2016 (accessed 27.09.2016).

[9] S. Yuan, F. Ansari, X. Liu, Y. Zhao, Optic fiber-based dynamic pressure sensor for WIM system, Sens. Actuators, A 120 (1) (2005) 53-58.

[10] P. Wierzba, B.B. Kosmowski, Polarimetric sensors for weigh-in-motion of road vehicles, Opto-electronics Reviw 2 (2000) 181-187.

[11] Intercomp, Improving vehicle data with WIM technology, Technology Profile, Traffic Technology International Magazine, inquiry no. 506 (2015) 100-101.

[12] SETRA-LCPC, Guide Technique: French design manual for pavement structures, SETRA and LCPC, Paris, May 1997.

[13] Itech-ALIZE, ALIZE-LCPC Software, User manual, Version 1.3, http://www. itech-soft.com/ALIZE/download/en/ALIZE-MU-V130-GB.pdf, (accessed 16.10.2016) 2011.

[14] D.M. Brumister, The theory of stress and displacement in layered systems and application to the design of airport runways, Proc. Highway Res. Board 23 (1943) 126-148.

[15] D. Duhamel, A. Chabot, P. Tamagny, L. Harfouche, ViscoRoute: visco-elastic modeling for asphalt pavements. Bulletin des Laboratoires des Ponts et Chaussées, pp. 258-259, 89-103 (2005).

[16] C. Huet, Etude par une méthode d'impédance du comportement viscoélastique des matériaux hydrocarbonés, Université de Paris, France, 1963. Ph.D. thesis.

[17] G. Sayegh, Contribution à l'étude des propriétés viscoélastiques des bitumes purs et des bétons bitumineux, Faculté des Sciences de Paris, France, 1965. Ph. D. thesis.

[18] A. Chabot, O. Chupin, L. Deloffre, D. Duhamel, ViscoRoute: a tool for the simulation of moving load effects on asphalt pavement, Road Materials and Pavements Design, Volume X, France 2009.

[19] C. Helg, L. Pfohl, Signal processing requirements for WIM Lineas Type 9195 Lineas Type 9195 documentation. Kistler Instruments AG., Winterthur, Switzerland, 2008.

[20] AFNOR, Dimensionnement structurel des chaussées routières - Application aux chaussées neuves, NF P98-086, october 2011. 